\title{
What are the Experiences and Needs of Primary Care Nurses in Caring for Patients With Type 2 Diabetes in a Rural Village in South Africa? An Exploratory Study
}

\author{
Ednah V Sechabe ${ }^{1}$, Tebogo M Mothiba ${ }^{1} \&$ Hilde Bastiaens ${ }^{2}$ \\ ${ }^{1}$ Department of Nursing Science, University of Limpopo, Sovenga, South Africa \\ ${ }^{2}$ Department of Primary and Interdisciplinary Care, Faculty of Medicine and Health Sciences, Universiteit \\ Antwerpen, Antwerpen, Belgium \\ Correspondence: Ednah V Sechabe, Department of Nursing Science, University of Limpopo, Private Bag X1106 \\ Sovenga 0727, South Africa. Tel: 27-82-313-7983. E-mail: evsechabe@vodamail.co.za
}

Received: April 18, 2019, Accepted: May 12, 2019, Online Published: June 11, 2019

doi:10.5539/gjhs.v11n7p90 URL: https://doi.org/10.5539/gjhs.v11n7p90

\begin{abstract}
Since 1994, the emphasis in the provision of health services in South Africa has shifted from hospital-based care to a community-based comprehensive primary health care system, especially important in the management of chronic diseases. However, primary health care professional nurses are not well trained to manage chronic conditions like type 2 diabetes. Therefore, this study aimed to explore the experiences and needs of primary care nurses as a basis for the development of a training programme for professional nurses who care for T2D patients. A qualitative descriptive approach was employed, using individual interviews with primary health care nurses caring for T2D patients in the Ga-Dikgale village clinics. Ethical considerations were observed throughout the study and quality supportive measures were employed. Three main themes emerged from the study findings which address the current practices and knowledge of professional nurses related to care provided to diabetes patients, the challenges experienced by professional nurses during the provision of care to diabetes patients on treatment and their training experiences, gaps and needs. A need for continuing education for professional nurses related to the care of patients with diabetes was identified. The results of this study will be used to develop a training programme to improve the knowledge and skills of professional nurses and to improve the quality of care of patients with type 2 diabetes.
\end{abstract}

Keywords: non-communicable diseases, T2D, training needs, training programme, primary care nurses

\section{Abbreviations}

T2D: Type 2 Diabetes; NCDs: Non-Communicable Diseases; PHC: Primary Health Care; HIV: Human Immunodeficiency Virus; TB: Tuberculosis; TREC: Turfloop Research Ethics Committee; EDL: Essential Drug List.

\section{Background}

Type 2 diabetes (T2D) is one of the fastest growing chronic non-communicable diseases (NCDs) and has become a global epidemic (Unnikrshnan, Pradeepa, Joshi, \& Mohan, 2017). Type 2 diabetes is characterized by impaired insulin secretion and insulin resistance in peripheral tissues, such as adipose and muscle, and the liver. The decrease in insulin secretion is due to the gradual decline in pancreatic beta-cell function (Stumvoll, Goldstein, \& van Haeften, 2005). Over the past three decades, the number of people with diabetes mellitus has more than doubled globally, making it one of the most important public health challenges for all nations (Chen, Magliano, \& Zimmet, 2011). Diabetes has caused 1.5 million deaths in 2012, an estimated 422 million adults were living with diabetes in 2014 (WHO, 2016). In 2013, about two-thirds of all individuals with diabetes lived in lower-middle-income countries (IDF, 2013). According to Braun and Clarke (2006), an estimated 415 million people have diabetes throughout the world, with the number set to rise beyond 642 million within the next two decades. Over $90 \%$ of cases of diabetes mellitus are of T2D a form of diabetes (WHO, 2009).

South Africa, like many other countries, reports an increase in the prevalence of non-communicable diseases such as diabetes mellitus (Malan, Mash, \& Murphy, 2015. This burden of disease has led to increased workloads, 
overcrowding of health facilities and poor quality of care. Also, it has exerted a tremendous strain on human resources in the healthcare system, especially on those working at Primary Health Care (PHC) level (Tsolekile, Puoane, Schneiden, Levitt, \& Steyn, 2014). Professional nurses play an extremely important role in the management of patients with T2D in PHC settings. They are often the first healthcare team members to interact with patients and are called on to apply their knowledge, training, and skills to treat, follow-up, educate and motivate patients with diabetes about self-care and practical ways to achieve their treatment goals (Levich, 2011).

Training of nurses at PHC level is vital to ensure that they can contribute appropriately to the management of non-communicable diseases, including diabetes. Because of their central role in primary healthcare, they are well placed to support early diagnosis in diabetes. It is essential that efforts are made to diagnose diabetes early to prevent the complications and to begin early management interventions of the disease (Ramachandran, 2014). Early control of glycaemia and dyslipidaemia, together with regular examination for macrovascular and microvascular complications, with appropriate and timely interventions, are the only ways to prevent and reduce morbidity and mortality related to diabetes (Amod, Motala, Levitt et al., 2012).

Nurses play a critical role in empowering patients to better manage diabetes through self-care and improving the quality of life of these patients through providing them and their families with the required information and consultations (Vissarion, Malliarou, Theofilou, \& Zyga, 2014). Enhancement of the glycemic control and quality of life of those afflicted with diabetes requires the incorporation of education in the treatment modality, aiming to teach diabetics the methods by which they can live with and manage their disease daily (Burke, Sherr, \& Lipman, 2014). Previous research has shown that behaviour change counselling integrated into routine primary care is effective in assisting patients to change their risky behaviours (Murphy, Mash, \& Malan, 2016).

Lack of knowledge and inconsistent practice pertaining to T2D diagnosis, evaluation and management among professional nurses, long intervals between patient visits and limited consultation time, resulting from overwhelmingly heavy patient loads constitute major impediments to the attainment of diabetes-related goals of (Roumie, Elasy, Wallston, Pratt, Greevy, Liu, Alvarez, Dittus, \& Speroff, 2007). Educational programmes for nurses with a focus on reducing the risk factors of diabetes, monitoring and controlling blood sugar levels and diagnosing, preventing, and treating hypo and hyperglycemic states are required (Peimani, Tabatabaei, \& Pajouhi, 2010). Nurses should be able to: screen patients for early identification, recognise and initiate corrective measures for inadequate treatment regimes, help patients set and achieve therapeutic goals and assess diabetes-related complications as they arise (Levich, 2011).

Prior research in the Ga-Dikgale area reveals that nurses lack training in chronic disease management. The nurses attended supplementary training programmes on HIV, TB, Child Health but little on diabetes, hypertension, mental health or cardiovascular diseases. This indicates that there is a need for the training of professional nurses who care for patients with diabetes in the Ga-Dikgale village clinics. However, to develop a context-specific training programme for those nurses, their specific knowledge gaps and training needs need to be identified (Maimela, Van Geertruyden, Alberts, Modjadji, Meulemans, Fraeyman, \& Bastiaens, 2015).

Therefore, the objectives of the study are to:

- Explore and describe the experiences of professional nurses related to care provided to patients with Type 2 diabetes;

- Identify the training needs of professional nurses regarding Type 2 diabetes.

\section{Method}

The research reported on in this paper is part of a larger study which aimed at the development of a training programme for primary care nurses on T2D in rural clinics in South Africa. In the first stage, a situational analysis was conducted, employing a mixed method approach, making use of the complementarity of qualitative and quantitative research methods to get insight into the knowledge, experiences and training needs of these nurses (Fetters, Curry \& Creswell, 2013). This paper reports on the qualitative part of the research. A descriptive qualitative study approach was adopted to explore and understand the experiences and learning needs of nurses taking care of patients with diabetes in the Ga-Dikgale village from their perspective.

\subsection{Study Site}

The study was conducted in the rural area of the Ga-Dikgale village in Limpopo Province, South Africa. The Ga-Dikgale village is situated in the Capricorn District, which is approximately 50km northeast of the capital city of Limpopo Province, Polokwane. The study was conducted in the three primary health care clinics: Sebayeng, Seobi and Dikgale which provide comprehensive integrated health care to the community. 


\subsection{Study Population and Data Collection}

The population eligible for this study was primary health care nurses working in the three clinics and having at least two years' clinic experience caring for patients with diabetes. A total population of purposive sampling was used to select the participants because all 27 participants met the selection criteria.

Semi-structured, one-on-one interviews with an interview guide (see Appendix-) were used to explore and describe the knowledge and practices of the professional nurses caring for patients with diabetes in the Ga-Dikgale village clinics. A semi-structured guide was developed by ES (female professional nurse, who had basic training in qualitative research) in collaboration with TM (professional nurse, experienced qualitative researcher) and HB (medical doctor, experienced qualitative researcher). The guide consists of three main questions (Table 1). Interviews were conducted by ES, who is working as a hospital nurse caring for patients with diabetes in another province in South Africa. This meant that she did not know the study participants. In the preparatory phase of the research, ES was in contact with the head nurses of the different clinics. Permission was asked to approach the nurses to invite them to participate in the study and to conduct the interviews during their working hours at the clinic. The individual interviews were voice recorded and field notes were written for the aspects that could not be captured by the voice recorder. Probing questions were asked to clarify issues during the interview sessions. A total of 18 professional nurses who were available during data collection were interviewed. Eight professional nurses were on their rest days and one was on leave during data collection. Sufficiency of the collected data was checked to ensure credibility and the second round of data collection was conducted where a total of 23 professional nurses were interviewed until sufficient data were available for the study. Interviews took between 25 and 40 minutes.

Table 1. Interview guide main questions

What are your experiences with patients with diabetes?

What are the challenges you face in the care for patients with diabetes?

Do you feel the need to learn more about care for patients with diabetes? Please explain how. What would you like to learn?

\subsection{Data Analysis}

Data were analysed using a thematic method of data analysis, combining both deductive and inductive coding. The main interview questions were used as an initial coding frame. The researcher read and re-read the transcripts several times to be familiar with the entire body of data before continuing with the analysis. After that, descriptive coding was undertaken, labelling relevant pieces of text and producing a list of descriptive codes. Data was put back together in new ways, making connections between codes and grouping similar codes into broader themes and sub-themes. Further grouping of the emerging ideas into sub-themes was carried out using constant comparison. Ideas from additional interviews were added and coded interviews were reread to ensure that they were comprehensive. During the whole coding process, regular discussions were held with the research team (ES; TM; HB) to support trustworthiness (King \& Horrocks, 2010).

\subsection{Ethical Considerations}

Ethical clearance was obtained from the University of Limpopo Research Ethics Committee (TREC). The study ethical clearance reference number is TREC/37/2016: PG. Permission to collect data in the health care institutions was obtained from the Limpopo Province Department of Health Research Ethics Committee. Informed consent was obtained from all participants before the start of each unstructured interview session. The aim and nature of the study were explained to the participants before data collection and they were informed that results would be used for scientific purposes and might be published. The names of the participants were not used and numbers were allocated to each participant to ensure anonymity. Only the researchers have access to the data obtained to maintain confidentiality (Holloway \& Galvin, 2016). Interviews were conducted in private rooms away from distractions to ensure privacy.

\subsection{Results}

In total, 23 nurses were interviewed. Interviews took an average of 25 to 40 minutes. Characteristics of the participants are described in Table 2. 
Table 2. The organisation of the participants

\begin{tabular}{ll}
\hline Characteristics & Number \\
\hline Experience & 3 \\
$<5$ years & 9 \\
$5-10$ years & 5 \\
11 - 15 years & 4 \\
$16-19$ years & 2 \\
$\geq 20$ years & 18 \\
\hline Level of education & 5 \\
Diploma & \\
Baccalaureate Degree & \\
\hline
\end{tabular}

The interview data were grouped in three main themes (Table 3): Current practices and knowledge of professional nurses related to care provided to diabetes patients on treatment, Challenges experienced by professional nurses during provision of care to diabetes patients on treatment, training experiences and gaps and needs.

Table 3. Themes and sub-themes

\begin{tabular}{ll}
\hline Main themes & SuSub-themes \\
\hline & Nurses perform diverse tasks and roles \\
1. Current practices and knowledge of professional & Nurses' collaboration with other disciplines \\
nurses related to care provided to diabetes patients & There is a diverse level of knowledge among nurses \\
\end{tabular}

\section{Challenges related to the patient}

Problems related to denial and misconceptions

Problems related to patients' continued risky practices and non-adherence to recommended treatment

Patients' socio-economic context

2. Challenges experienced by professional nurses during Problems related to inadequate knowledge or self-management the provision of care to diabetes patients on treatment skills

\section{Challenges related to the organisation of health care}

Shortage and non-functionality of machines and the shortage of medication

Shortage of nurses and other services in the clinics

Shortage of resources like guidelines and training

Lack of attendance and unavailability of diabetes training programmes viewed as problematic in managing the condition

3. Training experiences, gaps and needs

What topics nurses want training in

How nurses want the training-

\section{Theme 1: Current Practices and Knowledge of Professional Nurses Related to Care Provided to Diabetes Patients}

Experiences with T2D nurses described during the interviews reveal that in practice they perform several different tasks in diagnosing, treatment follow up, educating patients, management of complications, referring patients for specialised care and emergency care. The nurse's role during the diagnosis of diabetes includes a thorough assessment of the patients, rapid and laboratory blood glucose tests and urine dipstick tests. If the 
diagnosis is confirmed, the patient is offered health education about lifestyle, diet, exercises, and then the patient is referred to a doctor for the initiation of treatment. After this, the patient is referred back to the clinic for follow up treatment and further support and care by the nurses.

"If the patient comes to the clinic for the first time we do an initial assessment of the patient and check the blood sugar and we find that it is high then we tell the patient to come the following day in the morning before eating. Then we do the fasting blood glucose but we start by doing the rapid test to check maybe the blood glucose of yesterday it was triggered by something" [nurse 3].

Minor complications of diabetes and some emergency cases are managed in the clinics and later referred to a hospital if there is a need, the complicated cases are referred to the hospital for specialised care.

"Normally when it is a newly diagnosed because this patient does not have treatment if the blood sugar is 20 and above we normally put a Normal Saline drip to reduce the blood sugar then we call the ambulance for the patient and we refer the patient to the hospital" [nurse 1].

According to participants, patients are referred to the next level of care, based on the signs and symptoms that they present with when consulting the clinic. The signs and symptoms that the patients present with include: high glucose levels, dizziness, dry mouths, blurred vision and others. When referring patients, the referral note should outline the reason for referral. The participants mentioned what should be included in the referral i.e., patient's history, findings from the assessment and tests carried out and medication that the patient received before referral.

"If there is a problem we refer them to the hospital, for example, if a patient says l usually feel dizzy or has blurred vision after taking medication we advise the patient to go to the hospital and see the doctor because we are not sure what it is the cause" [nurse 3].

"Eeh, the contents we talk about what you find to the patient, the history and even my assessment. Whether there is a dry mouth for an example. You indicate everything there also even indicating the blood sugar level and treatment given [nurse 13].

The findings reveal that multidisciplinary team services and resources are available at the clinics to support the health services rendered to patients with diabetes. The multidisciplinary teams include the doctor, dietician and the pharmacist. The services of the multidisciplinary team occur on different dates. They may be weekly, monthly or fortnightly. According to the participants, there are scheduled days for doctors to visit the clinics and see patients with diabetes and also to give support to professional nurses.

"I consult colleagues or send patients to doctors in the hospital. On Thursdays, we are having visiting doctors if we are having problems we refer difficult patients to them. We also consult doctors in the hospital and refer the patients" [nurse 18].

"With those type of patients, the pharmacist come and dispense insulin to them. For the patients that are not in, we take their insulin and store in the fridge" [nurse 4].

"If you see that he comes in the consulting room you ask a question to check if he understands what you have told them in the morning when giving health education and the dietician is there if you find that this patient needs to be referred, we refer him immediately to the dietician to be seen by the dietician" [nurse 2].

The clinics receive support from the laboratory services every day, except weekends and holidays and the pharmaceutical services every week. The nurses collect specimens from patients, and the laboratory services come and collect the specimens for testing and bring the results back. The pharmaceutical services from the hospital visit the clinics to dispense medication that is not stored in the clinic. This includes insulin injections and oral medication.

"Yes, National Health Laboratory Services (NHLS) is coming every day from Monday to Friday. Weekends they are not coming. If the patient comes during the weekend we encourage them to come early in the morning Monday" [nurse 3].

The level of knowledge differs among the participating professional nurses: some lack of knowledge while others are knowledgeable about diabetes.

"Normally if the patient is newly diagnosed we refer them to the diabetic nurse so that they should go for training regularly or they need to be health educated by a person who knows this condition [nurse 13].

\section{Theme 2: Challenges Experienced by Professional Nurses During Provision of Care to Diabetes Patients}

Several challenges were experienced and described by the participants, linked both to the patient and to the healthcare system. 


\section{Challenges related to the patient}

The findings reveal that professional nurses experience challenges during the provision of care to patients with diabetes. Some challenges affect the patients' blood glucose levels and lead to complications related to diabetes. The attitudes, behaviours and other personal characteristics of patients contribute to poor treatment outcomes, lack of adherence to treatment and complications related to the disease. These may include: denial and misconceptions, continued risky behaviour/non-adherence to advised treatment and lack of knowledge and self-care skills.

Denial and misconceptions displayed by the patients retard progress and lead to treatment failure. Sometimes patients, after being diagnosed with diabetes, go into denial and think that they have been bewitched. They go to the traditional and faith healers for help and stop following the medical advice and taking their medication.

"Before newly diagnosed patients can accept they start by having denial emotions first because you find that before sending that person to diabetic nurse when they come home or they come back to our facility they still have that high blood sugar until they adjust themselves they just continue with those" [nurse 4].

"In December an old man who was known diabetic came to the clinic limping and said I have consulted traditional healers and have told my children that $l$ was bewitched. In the clinic, we gave the old man antibiotics and dresses the wounds until they are healed. I told him that it is not witchcraft, it is because of the blood sugar. So I teach patients that if they develop some sores or wounds which takes time to heal it is not because they have been bewitched" [nurse 2].

"Some patients deny that they are diabetic and will not stick to the advice about diet. They will continue taking wrong food and abuse alcohol and when you give health education about the lifestyle they will tell you straight that they are not diabetic" [nurse 14].

The participants think that patients' continued risky behaviour and non-adherence to advised treatment lead to uncontrolled blood glucose levels and complications related to diabetes. Patients are taught what to eat and what not to eat, when to come for follow up visits and how to take their medication. However, they still adhere to the risky practices of not following the instructions of the health care professionals. This happens especially during social events and funerals when some patients with diabetes do not adhere to the medical advice that they have been given. This may lead to patients presenting with uncontrolled symptoms which may lead to complications.

"Some patients do not comply even if you try to teach them and they don't care what they eat and the other thing is that when you tell the patient that when the sugar is not controlled the doctor will change the treatment and prescribe both the insulin and the oral anti-diabetes treatment, you find that the patient does not want to use the injection because they are used to oral and it becomes difficult to manage the client and the sugar and you find that the sugar becomes high all the time" [nurse 10].

"Hmm, my experience is that diabetes is not like hypertension it is difficult to control and most patients they find it difficult to control the sugar level because of the food they eat. Here in the clinic, most patients come with high sugar level when you try to ask about the food they eat you find that there is a problem. They were told what to eat but sometimes they find it difficult to adhere to the diet" [nurse 12].

"When you take history they will tell you that maybe l went to a funeral or something like that and late whatever is there. They don't consider their condition, but at home, they can say l am not eating one two three but when they go to funerals or parties you find that they just take whatever is available" [nurse 1].

The findings reveal that the patients' socio-economic context may lead to difficulty in adherence to treatment and related medical advice. Lack of money for transport when referred to the hospital for further management by the doctor or other medical teams and lack of support from family members lead to poor adherence to treatment and medical advice.

"If the patient fails to collect her insulin during the collection day maybe on Thursday and he comes to the clinic to report that her insulin is finished you can send her to the hospital is a challenges because you find that she is telling you that l don't have money or l don't have somebody who will accompany me to the hospital" [nurse 2].

"You can give the patient referral and the patient will say l don't have money and then she has to stay home for a while until she got the money" [nurse 5].

"I think it might be the background because most people in this area most of the people are not working they just eat what they come across even if they are restricted according to their condition because it is what is available they just eat" [nurse 13].

Nurses indicated the importance of knowledge and self-management skills and the fact that some patients possess knowledge and skills regarding diabetes and others do not. Knowledgeable patients seem to take their medication as prescribed and they follow medical advice and make good progress. Their blood glucose levels on 
follow up visits are controlled and they do not show signs of non-adherence and complications of diabetes.

"They must know what is happening in their bodies. Sometimes you will hear a person saying l don't eat sugar or a person will think to say he has diabetes because he ate a lot of sugar. So we tell them that diabetes is not there because they ate a lot of sugar. There is something in the body that is not working well" [nurse 18].

"They know what to eat and what not to eat and even their lifestyle they understand that they are not supposed to maybe in case of shoes they have to put on loose shoes so that they don't have to damage their legs or they don't have to walk barefooted because if they are pricked by something like needle or pin the wound will not heal quickly. They take their treatment the way you have told them" [nurse 1].

Lack of knowledge regarding diabetes is a challenge. Sometimes it is related to the fact that some patients are old and are not able to follow the treatment as prescribed.

"Ya, I have been working in the clinic seeing diabetic patients on Thursdays. Most of the patients are old and they are not sure about diabetes. They don't have knowledge that is why they eat like any person. They don't choose what they eat. We teach them what to eat but they don't understand. Some say instead of putting sugar in the tea they put brown sugar because they believe brown sugar does not raise blood sugar. Otherwise, they come to the clinic for treatment" [nurse 2].

Participants observed that some patients lacked knowledge and understanding of diabetes, self-management strategies and treatment and complications. Nurses stress the importance of their role in educating people about diabetes and the fact that they do not always succeed in educating their patients.

"The things that are hindering the treatment of the diabetic patients l can say the clients that do not have insight concerning the condition, they usually do things that they are not supposed to do" [nurse 5].

"I am not sure maybe the patients do not understand when we teach them about diabetes. Maybe we are not giving them enough information I don't know. They forgot what we teach them, some of the patients come high HGT sometimes 19 and above. They claim to be taking the treatment regularly, they don't adhere to the education we give them about diet, sometimes you find that the sugar is either high or low" [nurse 6].

"My experience is that most of the patients are unable to adhere to the treatment especially males. They sometimes forget to take their medication as prescribed; they lack self-management strategies. When you try to find out the reasons for non-adherence you are told that they have forgotten" [nurse 2].

"Some of the patients they don't know how to use pen insulin and they don't want it they refuse it they just want the syringe one" [nurse 2].

\section{Challenges related to the organisation of care}

The study findings reveal that there are challenges related to the organisation of health care in Ga-Dikgale village clinics. There is a shortage of some of the equipment used for the care of patients with diabetes. This makes it difficult for nurses to function as expected. Some clinics are short of equipment like glucometers, glucostix and blood pressure machines which are necessary for the baseline monitoring of the patients. In some of the clinics, the equipment is available but is non-functional. According to the participants, it takes a long time to replace and repair the broken equipment. The challenges related to the organisation of health care delays the proper assessment and management of patients.

"Sometimes we also don't have the resources to care for the patients. Like for an example, the HGT machine become broken and we don't know what to do or the test strips are not there" [nurse 17].

"The challenges that we come across sometimes the machines are not working and the patient coming to the clinic are not tested for their glucose level that day. You just give the medication but you don't know how blood glucose today. If there is a problem we refer them to the hospital, for example, if a patient says l usually feel dizzy or has blurred vision after taking medication we advise the patient to go to the hospital and see the doctor because we are not sure whether it is hypoglycemia or hyperglycemia" [nurse 3].

The study findings revealed that there is sometimes a shortage of medication in the clinics which leads to poor patient outcomes, medication errors and delayed or cancelled care. Sometimes patients are sent home and advised to come for medication on certain dates.

"Ok, we care for diabetic patients every day and sometimes we are experiencing a shortage of diabetic treatment at our facility, for an example Glucophage but we do ask from other facilities" [nurse 9].

"The challenge is that sometimes we don't have medication for diabetes in the clinic. You find that we have got one or two and the other medication is not there. And sometimes the blood glucose machine is not working and it becomes difficult to measure the blood sugar" [nurse 10]. 
The study findings reveal that there is a lack of dieticians in some of the clinics. Patients from the clinics that do not have dieticians are referred to the hospital, or to the other clinics, to be seen by the hospital dietician.

"No, we don't have a dietician in the clinic. If we want to refer the patient to the dietician we just refer to the hospital or clinics with dieticians. But before then the doctors are the one who initiates treatment in the hospital then they have got the chance to send the patient to the dietician. Then the dietician will advise them about the diet" [nurse 4].

"Yes, but those who don't stick to the diet we see by the blood sugar level when they are coming to collect treatment you find that their sugar is recording high. We refer them again to the hospital to be seen by the dietician" [nurse 2].

Some participants confirmed that there is a lack of diabetes nurses and that they rely on the doctors to see the patients instead of referring them to diabetes nurses who are well informed in teaching the patients about diabetes.

"There is no one who is trained as a diabetic nurse. We are all the same" [nurse 2].

"I suggest that maybe if we can have a diabetic nurse, somebody who will go for training on diabetes to be a diabetic nurse" [nurse 1].

The clinics have guidelines and protocols that should be followed during the care of the patients but, due to the shortage of resources, nurses are unable to adhere to the available guidelines and protocols.

"Yes, we do have guideline but we don't have the equipment to monitor patients like blood pressure machine and glucometers. We tell patients to come back the following month for follow up and monitoring" [nurse 17].

Availability of teaching aids would assist patients to understand their condition better. One participant confirmed that the use of teaching aids when offering health education to patients makes them understand and take an interest in knowing more. Looking at teaching aids enables them to ask questions.

"There was a day when $l$ was teaching patients about diabetes and l saw them looking at me. I was using something like a book which l found from one of the doctors. I showed the patient a poster from the book and they were anxiously looking at the posters to show that they want to know more about diabetes. I showed them the pancreas and l told them that the organ l am showing you is found in your body. I wish to have posters so that l can use to show them where sugar comes from or a doll to use in the morning when we give health education to use and demonstrate with it. Like if you have posters you will be able to show them the organ that leads to diabetes. You will show them and touch the part that is sick in the body that makes a person to be diagnosed as having diabetes. If possible show them the pancreas and tell them how does this lead to diabetes" [nurse 2].

\section{Theme 3: Training Experiences, Gaps and Needs}

The study findings reveal that most participants had not received training about diabetes other than during their basic training to be professional nurses. In the three clinics, only one professional nurse had attended diabetes training. The training was arranged by a private services provider. Participants think that the scarcity of diabetes training programmes leads to mismanagement of patients. The participants do not have confidence in what they are doing when they take care of patients.

"I'm still applying the skill that I obtained at college when l was training as a professional nurse. I learned about diabetes when l was in college as a student nurse" [nurse 3].

"All along we have been mismanaging patients because of unavailability of training regarding diabetes and l guess the majority of professional nurses who are not informative enough the trend continue of mismanaging or mishandling patients" [nurse 6].

"At the clinic, we are not taught to prescribe diabetic treatment we rely on the doctors from the hospital. So we issue treatment that has been prescribed by the doctors" [nurse 16].

"If the patient is the first time attending the clinic then diagnosed with high blood sugar that is where we not sure whether is Type 1 or Type 2 unless the doctor diagnoses that patient. That is where we find that it is difficult" [nurse 17].

One participant confirmed receiving training in the form of a workshop on two occasions while employed as a professional nurse. According to the participant, the knowledge gained during the basic training courses helped understand the condition and manage the patients effectively. She is now able to feel for the patients because in the training they were made to act like a patient with diabetes. According to the participants, it is important to attend diabetes training courses to improve patient care.

"Yes, I attended a three-day course where we training to live like a diabetic. The whole of three days we were given Placebos in the form of sweets being tablets for us. And then we were supposed to eat like a diabetic, exercise like 
a diabetic, inject ourselves with insulin, take medication after having those meals. In the morning take the fasting blood glucose, eat then after eating, test blood glucose again and then after that take medicines. And then we have witnessed that it is difficult to live like a diabetic patient because the majority of the class we did not manage to have the doses correctly at the prescribed scheduled times, the other one was about us how to screen, prescribe and take care of the patients with diabetes at the Primary Health Care level" [nurse 17].

"I liked the courses on diabetes because they improved my work performance and patient care. The first training taught us how to feel for a patient and the second one was teaching us on how to manage a patient with diabetes in the Primary Health Care setting" [nurse 17].

Since they had started practising as professional nurses, they had not attended any workshop except for the sessions where they reminded each other of what they had learnt.

"Hmm, I think we are having more information that we received during the basic training about diabetes but we just want to be reminded and most of the time we are busy we don't provide the proper care but the information we have" [nurse 18].

"I'm still applying the skill that I obtained at college when l was training as a professional nurse. I learned about diabetes when l was in college as a student nurse" [nurse 2].

\section{Training Needs}

The participants confirmed the need to receive training about diabetes. Also, they mentioned other topics that they wanted to receive training on. According to the participants, training would build their confidence when providing care to patients. Currently, they rely on the Essential Drug List (EDL) provided by the Department of Health and are not conversant with changes and new treatment protocols.

"Yes, l feel there is a need to learn more about diabetes because we rely on EDL only. Since things are developing and we find that we still use the old regimen and not the recent treatment protocols" [nurse 10].

"Yes, I think there is a need. Because now we are using the information that we got from training and things are changing, they must keep us updated with the new developments about diabetes" [nurse 15].

Participants listed the different topics they needed to be trained on. The listed topics include anatomy and physiology of diabetes, identification and management of complications related to diabetes, assessment, how to assist patients to self-manage the disease, coping strategies, diet, exercise and treatment adherence.

"I want to know the management of the different types of diabetes and the type of people who are at risk of being diabetic; how to prevent diabetes; we need maybe more information about diabetic" [nurse 3].

"We want to know how to teach patients about diabetes, about diet and self-management of diabetes and how to self-inject insulin” [nurse 8].

"I would like to learn more about ehh, foot care in the diabetic patient, how to manage complications of diabetic patients especially the hypoglycaemic patients and others we need to learn more" [nurse15].

"Yes isn't when you teach you must start with the introduction and tell us what diabetes is, give the background and everything. You have to start with everything. Like the anatomically part of it, coming to lifestyle and treatment" [nurse 5].

Participants gave different opinions about what form their training should take. Some participants would prefer in-service training, while others would prefer the training to be in the form of a workshop. Further, the participants indicated that other categories of nurses should be trained about diabetes because patients are first seen by them when checking vital signs before they are seen by professional nurses or doctors.

"I prefer the training to be in the form of an in-service because it can be done at any time. Whoever is at work can be in-service and can in-service other staff" [nurse 9].

"The training should be in the form of a workshop and should be conducted in a central place and not in the clinics because in the clinics there is no space" [nurse 4].

"Yes, I was suggesting that when you conduct the training you much teach all the categories because when the patient initiates at the clinic they are seen by the nursing assistant and they must know what the patient is expecting so that is why I'm suggesting that all categories must be included" [nurse 7].

\section{Discussion}

Diabetes is one of the four priority non-communicable health problems and a leading cause of morbidity and mortality worldwide (Kakkar, 2016). In South Africa, nurses are regarded as the backbone of primary health care services where patients with all types of diseases and health problems, including diabetes, are seen before they go 
to the next level of care (Swart, Pretorius, \& Klopper, 2015). This article aims to present the findings of a qualitative study of the experiences and training needs of primary care nurses in caring for patients with diabetes and to indicate how these needs will inform the development of a training programme. The findings correspond with previous studies which find that, although professional nurses at Ga-Dikgale clinics are at the forefront in the care of patients with diabetes, they lack the knowledge and are poorly trained in the management of chronic diseases, including the management of diabetes (Maimela et al., 2015).

This study highlights the great need for training in diabetes management and the care of patients with diabetes. Study participants indicated that as the front-line health professionals they have a vital role to play in the initial management of patients with diabetes in primary health care settings. They are responsible for the administration of medication, giving care to patients with diabetes, educating patients about the diabetic treatment regimen and lifestyle changes and this is in line with previous research conducted by Uğur, Demir, \& Akbal, (2015). The study findings indicate that there is a need to improve the knowledge and practice of professional nurses, particularly in the care of patients with diabetes, through continuous training.

In South Africa, the basic nurse training curriculum includes training about non-communicable diseases including diabetes and hypertension. According to the Nursing Act (2005), nursing education and training across South Africa are responding to changing needs, developments, priorities and expectations in health and healthcare. Nurses need to be equipped with the knowledge and skills to improve the health and wellbeing of patients and their families.

The study findings indicate that inadequate knowledge and practices related to the care and treatment of patients with diabetes give rise to poor patient outcomes. Despite the increased number of patients with diabetes in Ga-Dikgale, the training of nurses on the care of these patients is not been considered effective. The majority of participants revealed that they only received training about diabetes while they were training to be professional nurses and, since their being employed as professional nurses, they have not attended any workshop or course on diabetes. The study conducted in the Ga-Dikgale village clinics by Maimela et al., (2015) reveals that nurses attended training on HIV, TB, Child Health but little on diabetes, hypertension, mental health or cardiovascular diseases. In the clinics, the visiting doctor's support and initiate treatment of the newly diagnosed patients and nurses refer all newly diagnosed patients to the doctor for the initiation of treatment because of their lack of knowledge on how to initiate treatment.

In South Africa, the structure of the healthcare system comprises different levels, from the most basic services at Primary Health Care (PHC) level to the most sophisticated at tertiary levels of care. A holistic approach in caring for patients with diabetes, including collaboration with other health care professionals, is needed (Ofori \& Unachukwu, 2014). The studies reveal that when there is a need, patients have to be referred to other health care professionals and other levels of care for further management. Patients are usually referred to using a letter which states the reasons for referral. This referral letter acts as a permission slip to allow the patient easy access to treatment by a specialist at the hospital level. As soon as the problem for which a patient was referred to is solved or under control, the patient moves back to the clinics.

Nurses at the primary level of care should know how to refer to the next level of care and what and how to write the referral note. Participants also mentioned that, as they lack knowledge of how to manage complications related to diabetes, they refer patients with complications to the hospital to be seen by the doctor. The participants feel that it is good for patients to be referred for major complications that cannot be handled at the clinic level but that in minor cases, the nurses should be empowered to handle those cases at the clinic level.

This study has highlighted that there are several misconceptions about diabetes. Even when patients do know and understand the biomedical origin of diabetes, some patients still attribute the cause of the disease to supernatural forces like witchcraft. This is in line with the study conducted by Hjelm \& Nambozi, (2008) and Rutebemberwa, Katureebe, Gitta, Mwaka and Atuyambe, (2013). The common belief that the disease is attributed to witchcraft often lead to poor adherence to prescription, increases the risk of relapse, poor therapeutic outcomes and needless mortalities (Mattke, Haims, Ayivi-Guedehoussou, Gillen, Hunter, Klautzer et al., 2011).

The study further reveals that nurses have the primary role of empowering patients to better manage diabetes through self-care, adherence to treatment and the prevention of complications. This corresponds to the findings of other studies (Tol, Alhani, Shojaeazadeh, Sharifirad, \& Moazam, 2015). According to Taruna, Juhi, Dhasmana, Harish, (2014); Brown \& Bussell, (2011) poor medication adherence seems to be a significant barrier to the attainment of positive clinical outcomes among diabetes patients. Patient non-compliance is not only limited to the failure to take medication, but also to the failure to make lifestyle changes, undergo tests or keep appointments with physicians (WHO, 2016).

The majority of the participants feel that training would empower them to form patient support groups where the 
patients would support, encourage and teach each other self-care skills, the eating of correct food, exercising, coming for follow up treatment and taking their medication as prescribed. According to WHO, (2009) empowering patients with diabetes to be active participants in their care, requires skill and knowledge on the part of the primary health care nurses. Professional nurses have a responsibility to teach patients about adapting to a balanced diet, weight control mechanisms, the symptoms of hypoglycemia and hyperglycemia and their approach, providing a balance between the food intake and the amount of daily medication and activity, daily examination of the feet for possible ulcers, controlling blood pressure and cholesterol, visiting a physician on a regular basis for eye examinations and renal function testing.

\section{Implications for Practice}

The study findings imply that the only way for the patients to receive quality comprehensive care is to empower nurses through training to update their knowledge and practice related to diabetes. The Department of Health should acknowledge and support the need for continuous training of nurses in the PHC sector as the priority to enhance the knowledge and skills of the nurses. Training may be in the form of workshops and in-service training, formal or informal. Options for incorporating other categories of nurses into the training should be explored as they have a key responsibility to teach patients about the disease.

Trends in diabetes management are constantly changing and so nurses should be trained to acquire new information and new ways of caring for the patients with the disease. New guidelines and protocols to support knowledge and practice should be made available and their implementation should be monitored by the Department of Health. Continuous follow-ups and evaluation of the services provided by professional nurses should be conducted. Availability of essential functional medical equipment for patient care in the PHC clinics should be ensured. Lack of knowledge and non-functional equipment in the PHC facilities leads to compromised nursing care of patients with diabetes. On the positive side, nurses indicated that they consult colleagues and the doctors in the hospital if they encounter problems related to patient care. They also use EDL. The clinical guideline is available in the clinics for reference during the management of patients with diabetes. However, this also illustrates the lack of ('ready to use') knowledge, although it does indicate a relevant coping strategy to increase knowledge.

\subsection{Strengths and Limitations}

Total purposive population sampling was performed the researchers were able to gain insight into practices and needs from all but one nurse from the three clinics. This gives a good basis to tailor the training to specific needs.

At the same time, the limitation of the study to the three clinics of the Ga-Dikgale village is a weakness. However, a thick description of the study context and the explicit quotes support other researchers in judging the transferability of the results. It was difficult to recruit the participants for the interviews because of their workload in the clinics, and some managers were not willing to release the participants. Some of the interviews were short and the researcher had to conduct a second-round data collection session to reach sufficiency.

\section{Conclusion}

In conclusion, the study reveals that the professional nurses who participated in this study had diverse responsibilities related to patient care at the primary health care level. They have a certain level of knowledge of diabetes, however, some areas still need to be improved to enhance the quality of their care. As a result, continuous training to strengthen skills and knowledge in those domains is necessary. Training in the form of workshops, in-service training and refresher courses should be provided for all categories of nurses to prevent mortalities related to the disease. Nurses should be trained in managing newly diagnosed patients by initiating treatment, educating them on self-care, the importance of adherence to treatment, diet, lifestyle and coping strategies for people living with the disease.

Also, improved collaboration with other health professionals and the provision of functional equipment for use in the care of patients is important to improve the standard of care. A multidisciplinary team approach to care improves patient clinical outcomes through the development of an agreed treatment plan. Furthermore, it shortens the timeframes from diagnosis to treatment.

Lack of knowledge and non-functional equipment not only lead to poor health outcomes but also cause frustration in nurses. There is a need to support professional nurses in the primary health care setting by providing functional medical equipment and making sure that medication is always available.

\section{Ethics Approval and Consent to Participate}

This study was approved by the University of Limpopo Ethics Committee (Turfloop Ethics Research Committee). Permission to access the clinics was obtained from the Limpopo Provincial Research Committee. 


\section{Availability of Data and Material}

Not applicable.

\section{Authors' Contributions}

ES developed the study, collected and analysed the data and drafted the manuscript. HB and TM co-developed the study, assisted in data collection, participated in data analysis and contributed to the writing of the paper. All authors approved the final manuscript.

\section{Acknowledgements}

The authors gratefully acknowledge the Global Health Institute and the Department of Primary and Interdisciplinary care from the Universiteit Antwerpen and the University of Limpopo for the support and contributions to the study.

They also thank the Limpopo Department of Health for the permission to conduct the study, the clinic managers and the professional nurses from the Ga-Dikgale village clinics for participating in this study.

\section{Funding}

The author(s) gratefully acknowledges the financial support of the Flemish Interuniversity Council (VLIR UOS Limpopo project), Grant Number: ZIUS2018AP021.

\section{Competing Interests Statement}

The authors declare that they have no competing interests.

\section{References}

Amod, A., Motala, A., Levitt, N. et al., (2012). The 2012 SEMDSA Guideline for the Management of Type 2 Diabetes. JEMDSA, 17(1), S1-S94. https://doi.org/10.1080/22201009.2012.10872277

Braun, V., \& Clarke, V. (2006). Using thematic analysis in Psychology. Qualitative Research in Psychology, 3 : 77-101. https://doi.org/10.1191/1478088706qp063oa

Brown, M. T., Bussell, J. K. (2011). Medication Adherence: WHO Cares? Mayo Clin Proc., 86(4), 304-314. https://doi.org/10.4065/mcp.2010.0575

Burke, S. D., Sherr, D., \& Lipman, R. D. (2014). Partnering with diabetes educators to improve patient outcomes. Dovepress. https://doi.org/10.2147/DMSO.S40036

Chen, L., Magliano, D. J. \& Zimmet, P. Z. (2012). The worldwide epidemiology of type 2 diabetes mellitus-present and future perspectives. Nature Reviews Endocrinology, 228-236. https://doi.org/10.1038/nrendo.2011.183

De Vos, A. S., Strydom, H., Fouche, C. B., \& Delport, C. S. L. (2012). Research at Grassroots: For the Social Sciences and Human Services Professions. Van Schaik Publishers: Pretoria. South Africa.

Hjelm, K., \& Nambozi, G. (2008). Beliefs about health and illness: A comparison between Ugandan men and women living with Diabetes Mellitus. Int Nurs Rev., 55, 434-41.

Holloway, I., \& Galvin, K. (2016). Qualitative Research in Nursing and Healthcare (4th ed.). Wiley-Blackwell.

IDF. (2013). Diabetes Atlas. International Diabetes Federation (6th ed.). Brussels, Belgium: International Diabetes Federation.

Kakkar, R. (2016). The rising burden of Diabetes-Public Health Challenges and way out. Nepal J Epidemiol, 6(2), 557-559. https://doi.org/10.3126/nje.v6i2.15160

King, N., \& Horrocks, C. (2010). Interviews in Qualitative Research. SAGE. Thousand Oaks, California.

Levich, B. R. (2011). Diabetes management: optimizing roles for nurses in insulin initiation. J Multidiscip Healthc, 4, 15-24. https://doi.org/10.2147/JMDH.S16451

Maimela, E., Van Geertruyden, J., Alberts, M., Madjadji, S. E. P., Meulemans, H., Fraeyman, J., \& Bastiaens, H. (2015). The perceptions and perspectives of patients and health care providers on chronic diseases management in rural South Africa: a qualitative study. BioMed Central Health Services Research, 15, 143. https://doi.org/10.1186/s12913-018-3085-y

Malan, Z., Mash, B., \& Murphy, K. E. (2015). Development of a training programme for primary care providers to counsel patients with risky lifestyle behaviours in South Africa. African Journal of Primary Health Care Family Medicine, 7(1), 819. https://doi.org/10.4102/phcfm.v7i1.819

Mattke, S., Haims, M. C., Ayivi-Guedehoussou, N., Gillen, E. M., Hunter, L. E., Klautzer, L et al. (2011). 
Improving Access to medicines for non-communicable diseases in the developing world. In. Santa Monica, California: Rand Corporation

Murphy, K. M., Mash, R., \& Malan, Z. (2016). The case for behavioural change counselling for the prevention of NCDs and improvement of self-management of chronic conditions. South African Family Practice, 58(6), 249-252. https://doi.org/10.1080/20786190.2016.1187885

Nursing Act. (2005). Competencies - Primary Care Nurse Specialist. The Republic of South Africa.

Ofori, S. N., \& Unachukwu, C. N. (2014). A holistic approach to the prevention and management of type 2 diabetes mellitus in a family setting. Dovepress. http://dx.doi.org/10.2147/DMSO.S62320

Peimani, M., Tabatabaei, M. O., \& Pajouhi, M. (2010). Nurses' Role in Diabetes Care; A review. Iranian Journal of Diabetes and Lipid Disorders, 9, 1-9.

Rutebemberwa, E., Katureebe, S.K., Gitta, S. N., Mwaka, A. D., \& Atuyambe, L. (2013). Perceptions of diabetes in rural areas of Eastern Uganda. Curationis, 36(1), Pretoria Jan. 2013

Ramachandran, A. (2014). Know the signs and symptoms of diabetes. Indian J Med Res., 140(5), 579-581.

Stumvoll, M., Goldstein, B. J., \& van Haeften, T. W. (2005). Type 2 diabetes: principles of pathogenesis and therapy. Lancet, 365, 1333-1346.

Swart, R. P., Pretorius, R., \& Klopper, H. (2015). Educational background of nurses and their perceptions of the quality and safety of patient care. Curationis, 38(1), Pretoria 2015. http://doi.org/10.4102/curationis.v38i1.1126

Taruna, S., Juhi, K., Dhasmana, D. C., \& Harish, B. (2014). Poor adherence to treatment: A major challenge in diabetes. JIACM, 15, 26-9.

Tol, A., Alhani, F., Shojaeazadeh, D., Sharifirad, G., \& Moazam, N. (2015). An empowering approach to promote the quality of life and self-management among type 2 diabetic patients. J Educ Health Promot. 4(13). https://doi.org/10.4103/2277-9531.154022

Tsolekile, L. P., Puoane, T., Schneiden, H., Levitt, N. S., \& Steyn, K. (2014). The roles of community health workers in the management of non-communicable diseases in an urban township. African Journal of Primary Health Care and Family Medicine, 6(1). https://doi.org/10.4102/phcfm.v6i1.693

Unnikrishnan, R., Pradeepa, R., Joshi, S. R., \& Mohan, V. (2017). Type 2 Diabetes: Demystifying the Global Epidemic. Diabetes, 66(6), 1432-1442. https://doi.org/10.2337/db16-0766

Uğur, E., Demir, H., \& Akbal, E. (2015). Postgraduate education needs of Nurses' who are caregivers for patients with diabetes. Pakistan Journal of Medical Sciences, 31(3), 637-642. https://doi.org/10.12669/pjms.313.6732

Vissarion, B., Malliarou, M., Theofilou, P., \& Zyga, S. (2014). Improvement of Diabetic Patients Nursing Care by the Development of Educational Programs. Health Psychol Res., 2(1), 931. Published online 2014 Jan 13. https://doi.org/10.4081/hpr.2014.931

WHO. (2016). Global report on diabetes. WHO Press. Geneva. Switzerland.

WHO. (2009). Kenya, Country Cooperation Strategy at a glance, Retrieved from http://www.who.int/countryfocus/cooperation_strategy/ccsbrief_ken_en.pdf

\section{Copyrights}

Copyright for this article is retained by the author(s), with first publication rights granted to the journal.

This is an open-access article distributed under the terms and conditions of the Creative Commons Attribution license (http://creativecommons.org/licenses/by/4.0/). 